\title{
In-Hospital Death and Adverse Clinical Events in Elderly Patients According to Disease Clustering: The REPOSI Study
}

\author{
A. Marengoni, ${ }^{1}$ F. Bonometti, ${ }^{1}$ A. Nobili, ${ }^{2}$ M. Tettamanti, ${ }^{2}$ F. Salerno, ${ }^{3}$ S. Corrao, ${ }^{4}$ A. Iorio, ${ }^{5}$ M. Marcucci, ${ }^{5}$ \\ P.M. Mannucci, ${ }^{6}$ for the Italian Society of Internal Medicine (SIMI) Investigators ${ }^{*}$
}

\begin{abstract}
Objective: The aim of the study was to recognize clusters of diseases among hospitalized elderly and to identify groups of patients at risk of in-hospital death and adverse clinical events according to disease clustering. Method: This was a cross-sectional study conducted in 38 internal medicine and geriatric wards in Italy participating in the Registro Politerapie SIMI (REPOSI) study during 2008. The subjects were 1,332 inpatients aged 65 years or older. Clusters of diseases (i.e., two or more co-occurrent diseases) were identified using the odds ratios (OR) for the associations between pairs of conditions, followed by cluster analysis. Logistic regression models were used to evaluate the effect of disease clusters on in-hospital death and adverse clinical events.

Results: A total of $86.7 \%$ of the patients were discharged, $8.3 \%$ were transferred to another hospital unit, and $5.0 \%$ died during hospitalization; $36.4 \%$ of the patients had at least one adverse clinical event. Patients affected by the clusters, including heart failure (HF) and either chronic renal failure (CRF) or chronic obstructive pulmonary disease, had a significant association with in-hospital death (OR, 4.3;95\% confidence interval [CI], 1.6-11.5; OR, 2.9; 95\% CI, 1.1-8.3, respectively), as well as patients affected by CRF and anemia (OR, 6.1; 95\% CI, 2.3-16.2). The cluster including HF and CRF was also associated with adverse clinical events (OR, 3.5; 95\% CI, 1.5-7.8). The effect of both HF and CRF and anemia and CRF on in-hospital death was additive.

Conclusion: Several groups of older patients at risk of in-hospital death and adverse clinical events were identified according to disease clustering. Knowledge of the relationship among co-occurring diseases may help developing strategies to improve clinical practice and preventative interventions.
\end{abstract}

\section{Introduction}

D UE to THE AGING OF THE POPUlation, an increasing proportion of elderly persons are hospitalized in acute care units. In most cases, elderly inpatients are affected not only by the acute disease leading to hospitalization, but also by chronic conditions that complicate the hospital stay and make it difficult for the patients to cope with acute events. Clinical management of the acute diseases is challenging per se, but when the patients also suffer from multiple other disorders, the process of clinical decision-making becomes even more difficult and the hospital outcome more uncertain.
Thus, evaluating the outcome of older inpatients must take into account the confounding effect of patient comorbidity. ${ }^{1}$ Some studies developed or used indexes of co-morbidity to predict adverse outcomes related to hospitalization, showing that higher scores of co-morbidity indices are associated with in-hospital mortality. ${ }^{2,3}$ Major limitations of these indices are that they usually do not cover the broad spectrum of conditions affecting the patients, and they frequently assign the same score to diseases of different severity. Moreover, as the association of diseases in patterns of co-morbidities is naturally giving rise to few specific clusters, the power of any index of co-morbidity as a predictor of

\footnotetext{
${ }^{1}$ Geriatric Unit, Spedali Civili, Department of Medical and Surgery Sciences, University of Brescia, Brescia, Italy.

${ }^{2}$ Department of Neuroscience, Mario Negri Institute for Pharmacological Research, Milan, Italy.

${ }^{3}$ Medicina Interna, IRCCS Policlinico San Donato, Department of Medical and Surgery Sciences, University of Milano, Milan, Italy.

${ }^{4}$ Biomedical Department of Internal Medicine, University of Palermo, Palermo, Italy.

${ }^{5}$ Department of Internal Medicine, University of Perugia, Perugia, Italy.

${ }^{6}$ Department of Medicine and Medical Specialties, IRCCS Maggiore Hospital Foundation, Milan, Italy.

*The participating units and co-authors are listed in the Appendix.
} 
death is limited by the discrete number of association found in practice. $^{2}$ Other studies have analyzed the role of specific co-morbidities on adverse events during hospitalization and outcome at hospital discharge. Zekry and colleagues showed that patients affected by an acute condition plus dementia have a higher risk of being discharged to a nursing home. ${ }^{4}$ Other authors focused on the effect of different co-morbidities on the occurrence of in-hospital complications for index diseases such as diabetes and heart failure (HF), showing that there is a correlation between co-morbidity and complications of treatment. ${ }^{5}$ However, the overall effect of aggregation and co-occurrence of diseases, beyond that leading to hospitalization, on in-hospital death and adverse clinical events during hospital stay remains to be well elucidated.

The aim of this study was to recognize clusters of diseases in elderly Italian persons hospitalized in acute care wards and to identify which of these clusters are associated with the risk of in-hospital death and adverse clinical events during hospitalization.

\section{Methods}

\section{Data collection}

The present study was held between January, 2008, and December, 2008, in 38 hospitals located in different regions of Italy, all participating in the Registro Politerapie SIMI (REPOSI) study, a collaborative effort between the Italian Society of Internal Medicine (SIMI) and the Mario Negri Institute of Pharmacological Research. The REPOSI study was designed with the purpose of creating a network of internal medicine and geriatric wards to evaluate patients affected by multiple diseases and prescribed with polytherapy. Participation in the network was on a voluntary basis, but in the choice of the participating centers attention was given to their homogeneous composition in terms of geographic distribution, size, and unselected admissions from the territory or the emergency room. The specific aims of the REPOSI study were: To describe the prevalence of co-occurring multiple diseases and treatments in hospitalized elderly patients, to correlate clinical characteristics of the patients with type and number of diseases and treatments, and to evaluate the main clinical outcomes at hospital discharge. The study included two phases. Phase one was designed to create the network of internal medicine and geriatric wards, and phase two was intended to activate a registry of patients included in the study.

All of the patients admitted to the wards participating in the study were recruited consecutively if they were 65 years old or older. Participation in the study was voluntary and an informed consent was signed by all the patients. A sample of at least 40 patients consecutively admitted to each participating center during a period of 4 weeks, 3 months apart each from the other (one in February, one in June, one in September, and one in December, 2008) was included in the study. A standardized Web-based case report form was filled in by the attending physicians, including sociodemographic factors, clinical parameters, diagnoses and treatments at both hospital admission and discharge, clinical events during hospitalization, and outcome. All the data recorded in the net were collected and checked by a central monitor institution (the Mario Negri Institute for Pharmacological Research, Milan).

The initial study sample included 1,411 subjects. Of these, $79(5.6 \%)$ were excluded due to missing or incomplete data;
25 had missing data on hospital outcome and 54 on sociodemographic and clinical characteristics due to errors in data input and recording. Of these 54, $6(11 \%)$ were transferred to other hospital unit and 3 (5.5\%) died during hospitalization. Hence, 1,332 individuals were available for the present analyses.

\section{Assessment of diseases}

Diseases examined in this study were collected at hospital admission and confirmed by clinical examination, clinical history, and laboratory and instrumental data collected by the attending physicians. Diagnoses were made using standardized criteria. The International Classification of Diseases, Ninth Revision (ICD-9) ${ }^{6}$ was used for classifying all the diseases. Only diseases with a prevalence of at least 5\% $(n=19)$ were taken into account in this study. For the purpose of this study, clusters of diseases were defined as two or more co-occurring specific conditions, according to Boyd and colleagues. ${ }^{7}$ Drugs were registered according to the Anatomical Therapeutic Chemical (ATC) classification system. ${ }^{8}$

\section{Functional status}

A patient was defined as severely dependent if needing help in activities of daily living $24 \mathrm{~h}$ /day.

\section{Adverse outcome and clinical events}

Adverse clinical events were defined as any acute clinical problem that occurred during hospitalization. Adverse outcome during hospitalization was in-hospital mortality. Patients who were not discharged home $(n=111)$ were excluded due to lack of information about their final outcome. Of these, 6 were terminally ill at hospital admission and transferred to end-of-life care units, 44 were transferred to rehabilitation units or long-term facilities, and 61 were transferred to other hospital units for the onset of acute medical or surgical diseases during hospitalization.

\section{Statistical analysis}

Prevalence per 100 was estimated for each disease and disease pairs. Several logistic regression models were run to analyze the crude and adjusted bivariate association between each pair of co-occurring diseases. The adjustment included age (years, continuous), gender, and education (years of schooling, continuous). To avoid that the multiple significance testing between pairs of diseases would give a high probability of finding a significance difference just by chance, we limited our analyses to diseases with a prevalence $>5 \%$. Moreover, the $p$ value for significance was set to $<0.001$ or lower. To further analyze and confirm patterns of comorbidity, a cluster analysis was performed. A correlation matrix was computed among all the diseases using the Yule $Q$ measure of association and average linkage as combination method. Several logistic regression models were run to analyze the crude and adjusted association between all the identified clusters of diseases with in-hospital death and adverse clinical events during hospitalization (1+ vs. 0 events).

A logistic regression model was run to test if the association with adverse clinical events was confirmed after adding patients transferred to the other hospital unit for the onset of acute medical or surgical diseases. Finally, to test if any cluster of diseases had a synergistic effect on in-hospital 
TABle 1. Sociodemographic Characteristics of the Patients and Prevalence of Diseases

\begin{tabular}{|c|c|}
\hline & $\begin{array}{l}\text { Patient numbers }(\%) \\
\quad(\mathrm{n}=1,332)\end{array}$ \\
\hline Age, years, mean (SD) & $79.4(7.5)$ \\
\hline Females & $722(54.2)$ \\
\hline Education, years, mean (SD) & $6.3(3.7)$ \\
\hline Hypertension & $770(57.8)$ \\
\hline Diabetes mellitus & $320(24.0)$ \\
\hline CHD & $307(23.0)$ \\
\hline $\mathrm{AF}$ & $275(20.6)$ \\
\hline COPD & $267(20.0)$ \\
\hline CVD & $260(19.5)$ \\
\hline Malignancy & $198(14.9)$ \\
\hline Gastric diseases & $185(13.9)$ \\
\hline Dyslipidemia & $180(13.5)$ \\
\hline $\mathrm{CRF}^{1}$ & $170(12.8)$ \\
\hline Anemia & $135(10.1)$ \\
\hline Thyroid diseases & $127(9.5)$ \\
\hline $\mathrm{HF}$ & $113(8.5)$ \\
\hline Anxiety & $105(7.9)$ \\
\hline Prostate hypertrophy & $105(7.9)$ \\
\hline Liver cirrhosis & $100(7.5)$ \\
\hline Dementia & $99(7.4)$ \\
\hline Arthritis & $95(7.1)$ \\
\hline Intestinal diseases & $94(7.1)$ \\
\hline $\begin{array}{l}\text { Number of prescribed } \\
\text { drugs, mean (SD) }\end{array}$ & $4.7(2.8)$ \\
\hline Severe dependency & $97(7.3)$ \\
\hline $\begin{array}{l}\text { Number of clinical } \\
\text { events, mean (SD) }\end{array}$ & $0.6(1.2)$ \\
\hline At least one clinical event & $485(36.4)$ \\
\hline Discharged & 1155 (86.7) \\
\hline Died & $66(5.0)$ \\
\hline
\end{tabular}

$\mathrm{SD}$, standard deviation; $\mathrm{CHD}$, coronary heart disease; $\mathrm{AF}$, atrial fibrillation; COPD, chronic obstructive pulmonary disease; CVD, cerebrovascular disease; $\mathrm{CRF}$, chronic renal failure; $\mathrm{HF}$, heart failure. death and adverse clinical events, or if the association was mainly due to a single disease included in the cluster, further logistic regression models were run, each assessing the effect of only one clinical condition. Age, gender, education, number of drugs, and severe dependency were entered in all the models as covariates. All of the multivariate analyses were also adjusted for the participating centers with no statistically significant change in the results (data not shown). Finally, a post hoc analysis of the sample size showed that less than 1,310 patients would be needed for an outcome occurrence of $5 \% \pm 1.18 \%$ as the highest deviation. All of the statistical calculations were performed with the software STATA $9^{\text {th }}$ version (College Station, TX).

\section{Results}

Of the 1,332 patients that were included in the analyses, $54 \%$ were females. The mean age of the patients was 79.4 years (standard deviation $[\mathrm{SD}]=7.5$ ). The most frequent diagnoses at hospital admission were: Hypertension (57.8\%), diabetes mellitus $(24.0 \%)$, coronary heart diseases (CHD, 23.0\%), atrial fibrillation (AF, 20.6\%), chronic obstructive pulmonary disease (COPD, 20.0\%), and cerebrovascular diseases (CVD, 19.5\%). The average number of prescribed drugs at hospital admission was $4.7(\mathrm{SD}=2.8)$. Ninety-seven $(7.3 \%)$ of patients were classified as severely dependent. A total of $86.7 \%$ of the patients were discharged at the end of hospital stay; $8.3 \%$ of them was transferred to another hospital unit and 5.0\% died during hospitalization. In all, $36.4 \%$ of the patients had at least one adverse clinical event during the hospital stay (ranging from 0 to 9 events) (Table 1). The most frequent adverse events were urinary infection $(12.0 \%)$, fever $(6.0 \%)$, anemia $(5.2 \%)$, pneumonia $(5.0 \%)$, electrolyte disorders $(4.5 \%)$, AF $(4.3 \%)$, heart failure $(3.0 \%)$, and acute renal failure $(2.7 \%)$.

Table 2 shows the results of the logistic regression analyses aimed at identifying associations between pairs of

Table 2. Odds Ratios (OR) and 95\% Confidence Intervals (95\% CI) for the Association Between Pairs of Diseases. Number $(N)$ of Cases, Deaths, and Events are Reported

\begin{tabular}{|c|c|c|c|c|c|}
\hline & $\mathrm{n}$ & $\mathrm{n}$ & $\mathrm{n}$ & OR $(95 \% \mathrm{CI})$ & OR $(95 \% \mathrm{CI})$ \\
\hline Pairs of diseases & Cases & Deaths & Events & Crude & Adjusted $^{\mathrm{a}}$ \\
\hline Hypertension and diabetes & 214 & 6 & 89 & $1.6(1.2-2.1)$ & $1.7(1.3-2.2)$ \\
\hline Hypertension and CVD & 183 & 5 & 70 & $1.9(1.4-2.6)$ & $2.0(1.4-2.7)$ \\
\hline Hypertension and dyslipidemia & 151 & 1 & 48 & $4.5(2.9-6.8)$ & $4.5(2.9-6.8)$ \\
\hline $\mathrm{HF}$ and $\mathrm{AF}$ & 60 & 7 & 31 & $5.2(3.5-7.8)$ & $4.6(3.1-7.0)$ \\
\hline $\mathrm{HF}$ and COPD & 41 & 6 & 22 & $2.4(1.6-3.6)$ & $2.2(1.5-3.5)$ \\
\hline HF and CRF & 32 & 21 & 7 & $3.0(1.9-4.8)$ & $2.4(1.5-3.9)$ \\
\hline CHD and COPD & 84 & 7 & 39 & $1.7(1.3-2.3)$ & $1.6(1.2-2.2)$ \\
\hline Diabetes and CHD & 118 & 4 & 50 & $2.5(1.9-3.3)$ & $2.5(1.9-3.3)$ \\
\hline Diabetes and CVD & 79 & 4 & 34 & $1.5(1.1-2.0)$ & $1.7(1.2-2.3)$ \\
\hline Diabetes and dyslipidemia & 69 & 1 & 25 & $2.2(1.6-3.1)$ & $2.0(1.4-2.9)$ \\
\hline Diabetes and CRF & 65 & 6 & 33 & $2.2(1.5-3.0)$ & $2.3(1.6-3.3)$ \\
\hline Liver cirrhosis and anemia & 20 & 1 & 7 & $2.4(1.4-4.1)$ & $2.9(1.7-4.9)$ \\
\hline Liver cirrhosis and malignancy & 33 & 1 & 3 & $3.2(2.0-4.9)$ & $2.9(1.8-4.7)$ \\
\hline Thyroid dysfunction and AF & 43 & 4 & 15 & $2.1(1.4-3.1)$ & $2.5(1.6-3.8)$ \\
\hline CRF and anemia & 32 & 7 & 16 & $2.4(1.5-3.7)$ & $2.6(1.7-4.1)$ \\
\hline Gastric and intestinal diseases & 29 & 0 & 13 & $3.1(1.9-4.9)$ & $3.1(1.9-5.0)$ \\
\hline
\end{tabular}

${ }^{a}$ Adjusted for age, gender, and education.

$\mathrm{CVD}$, cerebrovascular disease; $\mathrm{HF}$, heart failure; $\mathrm{AF}$, atrial fibrillation; $\mathrm{COPD}$, chronic obstructive pulmonary disease; $\mathrm{CRF}$, chronic renal failure; $\mathrm{CHD}$, coronary heart disease. 


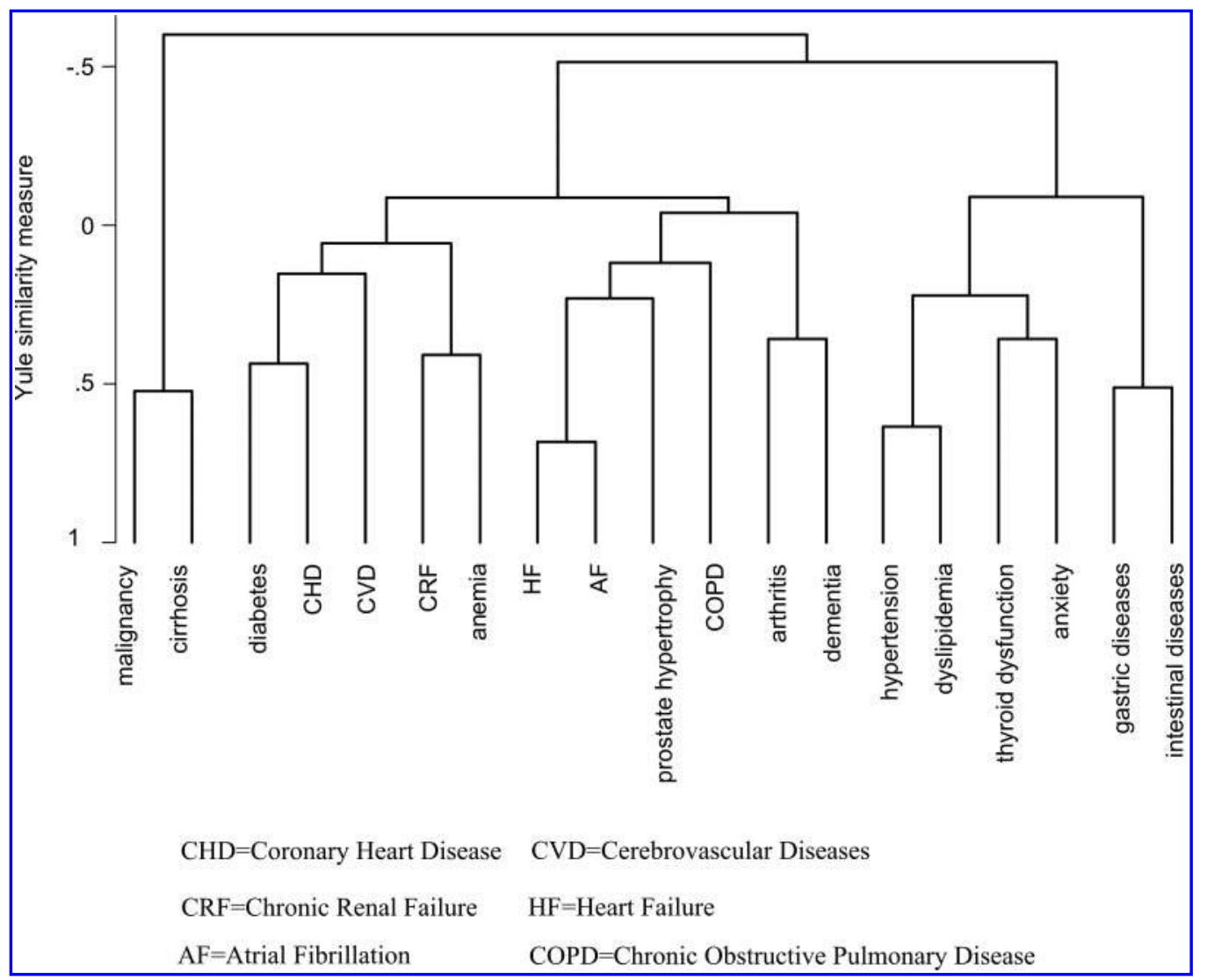

FIG. 1. Dendrogram resulting from cluster analysis testing the distribution and aggregation of diseases in the patients. Yule similarity is a measure of association. CHD, Coronary heart disease; CVD, cerebrovascular diseases; CRF, chronic renal failure; HF, heart failure; $\mathrm{AF}$, atrial fibrillation; COPD, chronic obstructive pulmonary disease.

diseases, as well as the number of in-hospital deaths and adverse clinical events for each pair of conditions. The logistic regression models were adjusted for age, gender, and education. Several pairs of co-occurring diseases emerged as associated: Only the statistically significant odds ratios (ORs) are reported. Beyond the expected connection between several cardiovascular conditions, two endocrine diseases (diabetes mellitus and thyroid dysfunction) clustered with other diseases such as dyslipidemia, chronic renal failure (CRF), and CVD. Liver cirrhosis was also significantly associated with both anemia and malignancy. Finally, COPD showed a correlation with two cardiovascular conditions: HF and CHD; CRF with anemia; and gastric diseases with intestinal diseases (Table 2). The aggregation of diseases was further tested by using cluster analysis, and eight clusters were identified. Four of them confirmed the associations between previously identified pairs of diseases, as one included liver cirrhosis and malignancy, one CRF and anemia, one hypertension and dyslipidemia, and one gastric and intestinal diseases. Two clusters included two other pairs of diseases: Dementia and arthritis and thyroid dysfunction and anxiety. Finally one cluster included three conditions-diabetes mellitus, CHD, and CVD-and one cluster included four conditions- $\mathrm{HF}$, $\mathrm{AF}$, and COPD together with prostate hypertrophy (Fig. 1).

Figure 2 shows the ORs for in-hospital death and adverse clinical events during hospitalization in relation to diseases clusters. Patients affected by CRF and anemia had an increased mortality during hospitalization (OR, 6.1; 95\% CI,
2.3-16.2). The clustering of HF with COPD (OR, 2.9; 95\% CI, 1.1-8.3) or CRF (OR, 4.3; 95\% CI, 1.6-11.5) also emerged as associated with in-hospital death. In addition, the clustering of HF with CRF (OR, 3.5; 95\% CI, 1.5-7.8) showed an association with adverse clinical events (Fig. 2). No other clusters of diseases emerged as significantly associated with inhospital death or adverse clinical events. When patients transferred to other hospital units for the onset of acute medical or surgical diseases were included in the analysis of adverse events, the association of HF with CRF and adverse clinical events was confirmed (OR,3.1; 95\% CI, 1.4-6.9).

To test if clusters of diseases had a synergistic effect on in-hospital death and adverse clinical events, or if the association was mainly due to a single disease, further logistic regression models were run, each assessing the effect of the single disease included in the clusters. Only three single diseases were significantly associated with the outcome of interest. HF and CRF were associated with both in-hospital death (OR, 2.1; 95\% CI, 1.0-4.3, and OR, 3.1; 95\% CI, 1.6-5.8, respectively) and adverse clinical events (OR, 1.5; 95\% CI, 1.0-2.2, and OR, 1.6; 95\% CI, 1.1-2.3, respectively), whereas anemia was associated only with in-hospital death (OR, 2.4;95\% CI, 1.2-4.8) (Fig. 2).

\section{Discussion}

In this study carried out in hospitalized elderly patients, we examined the co-occurrence of diseases by using the 


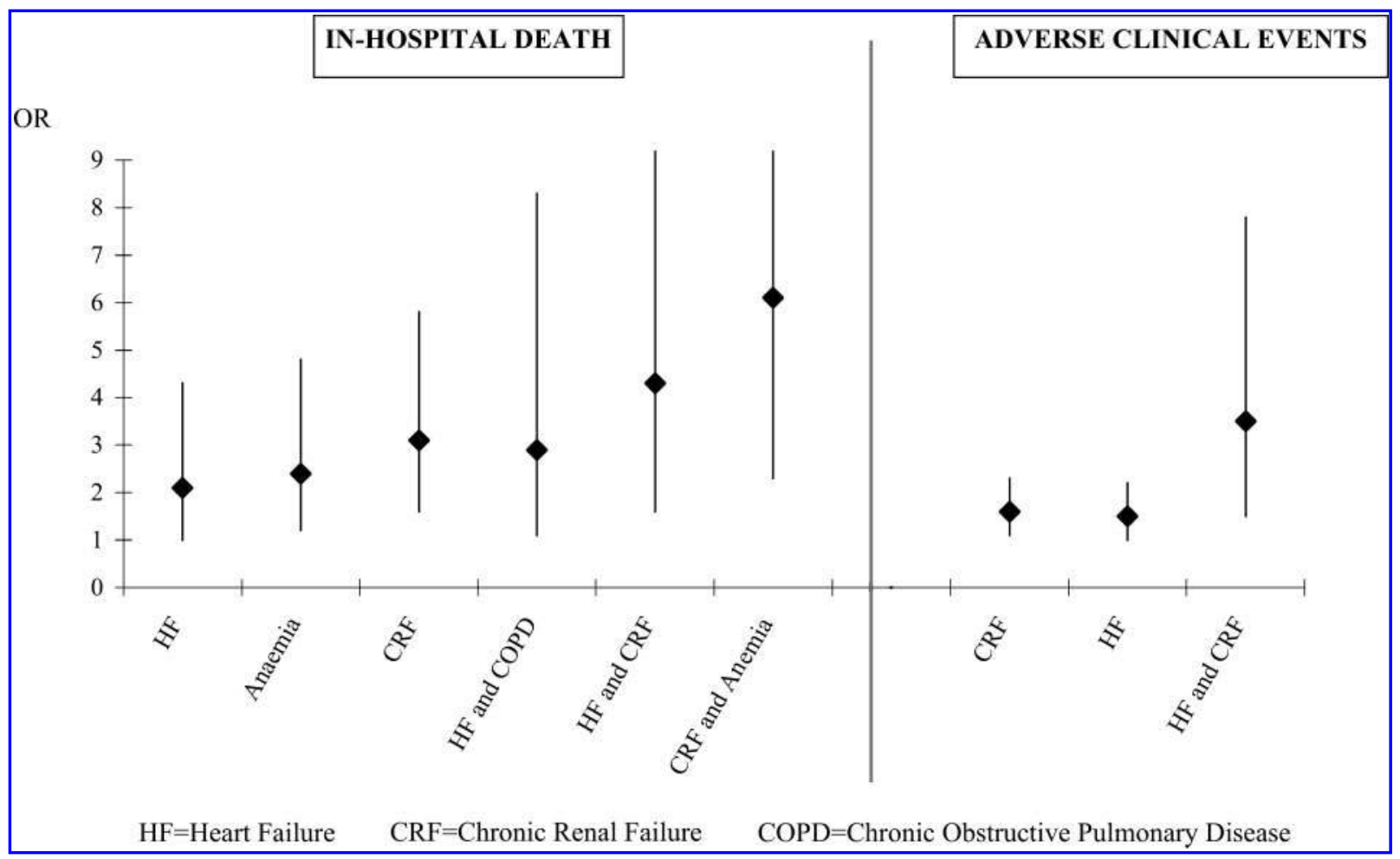

FIG. 2. Odds ratio (OR) and 95\% confidence intervals for in-hospital death and adverse clinical events during hospitalization due to different clusters of diseases. Models adjusted for age, gender, education, number of drugs, and severe dependency. HF, Heart failure; CRF, chronic renal failure; COPD, chronic obstructive pulmonary disease.

cluster approach. We found that some definite clusters of clinical conditions were associated with in-hospital death and adverse clinical events during hospital stay. First, we analyzed clustering of diseases by using the OR as a measure of the relative association between pairs of conditions. This approach is useful in assessing the degree to which comorbid diseases exceed a level expected by chance alone. ${ }^{9}$ Conditions that co-occur can have either a noncausal statistical association or share a common pathophysiological cause. ${ }^{10}$ Several clusters of diseases were identified; CVDs commonly occurred together, and showed a significant association with diabetes, CVD, CRF, COPD, dyslipidemia, and thyroid dysfunction. Diabetes, CRF, anemia, liver cirrhosis, and malignancy were also associated with each other.

An alternative approach to addressing the association between diseases is to use cluster analysis, a descriptive technique that considers how variables tend to occur in conjunction with each other. With this method, it is possible to go beyond simple co-morbid pairs to obtain a general overall pattern of how diseases are associated in a particular population and to establish where a particular disease of interest appears in the pattern. The assumption of the cluster method is that the distribution of diseases seen in the cluster should be significantly different from the distribution by chance. Cluster analysis identified eight clusters: A cardiopulmonary cluster (comprehensive of $\mathrm{HF}, \mathrm{AF}$, and COPD, together with prostate hypertrophy); a metabolic cluster (hypertension and dyslipidemia); four clusters made of a disease and its possible consequences (liver cirrhosis and malignancy; CRF and anemia; diabetes mellitus, CHD, and CVD; and thyroid dysfunction and anxiety); one compre- hensive of dementia and arthritis; and one including gastric and intestinal diseases.

To the best of our knowledege this is the first study evaluating aggregation of diseases in hospitalized elderly by using cluster analysis. Only a few previous studies that were conducted outside hospitals have used this method. For instance, John and colleagues used cluster analysis to describe the distribution of diseases in a sample of elderly American Indians ${ }^{11}$ and found that diseases aggregated in two major clusters, i.e., the cardiopulmonary and the sensory motor one. In another study held in a Swedish community-living population, five clusters of chronic diseases were identified, showing an association among cardiovascular and neuropsychiatric diseases. $^{12}$

Patients affected by specific cluster of diseases had different rates of mortality and clinical events during hospitalization. The clustering of HF with COPD or CRF and that of CRF with anemia were associated with in-hospital death; the coexistance of HF with CRF also showed an association with adverse clinical events. When the association of the single diseases included in the different clusters was analyzed in terms of in-hospital death, an additive effect between HF and CRF and between CRF and anemia was found.

These findings are difficult to compare with those of other studies, due to varied methodologies and settings. Despite several studies that have attempted to address the consequences of co-morbidities for different aspects of health care-such as in-hospital complications, outcome, costs, and length of stay-the majority of them were based on acute index disease leading to hospitalization and their comorbidities, or on patients admitted to a single hospital. ${ }^{13}$ At 
variance with these studies, the REPOSI study did evaluate the effect of the most prevalent diseases and their clustering on adverse hospitalization, irrespective of the cause of hospital admission. In spite of the fact that the use of disease clusters may overlook the effects of other health conditions and does not exclude that in-hospital death and adverse events depended upon the main disease, knowledge of the distribution and patterns of diseases in a hospitalized population may help to develop specific strategies meant to improve clinical practice and prevention. It has been shown that the risk of avoidable complications during hospitalization is strongly correlated with number of diseases. ${ }^{14} \mathrm{How}$ ever, to go beyond the arithmetical sum of coexisting diseases, and in view of the fact that individual diseases differ in their prognosis and interaction, we identified different subgroups of patients at risk of adverse hospitalization.

Observations of synergistic effect of specific combinations of diseases on in-hospital death and adverse events are important for several different aspects. First, for individual patient care, a larger benefit may be achieved by early recognition and adequate treatment of co-morbid conditions, as well as by intensively monitoring patients with co-morbidities. Currently, in older adults with multiple diseases, disorders not designated as the primary condition are often undertreated. ${ }^{15}$ Moreover, evidence-based medical treatments are usually directed toward single diseases, yet ignoring that concomitant diseases may lead to harm. ${ }^{16}$ Indeed, the main advantage of our approach is that not only it does identify patients at risk but also the specific diseases that by clustering together contribute to increase patient risk. A closer control and treatment of such diseases may help to decrease adverse outcomes inpatients at risk. Second, these results are important for an interdisciplinary approach, because they suggest the need of involvement and coordination among several health-care professionals (i.e., internists and geriatricians along with other specialists and general practitioners). Finally, these observations may be relevant for research purposes, because the evidence that disease A clusters with disease B could suggest new pathophysiological correlations or disease-related susceptibility.

Two major strengths of the REPOSI study are the multicenter design that involved 38 internal medicine and geriatric wards throughout Italy, resulting in a sample representative of the elderly hospitalized population of the country, and the inclusion of the patients during a period of 4 weeks (one per season) to balance the common effect of seasons on the acute diseases leading to hospitalization. However, a few limitations must also be mentioned. First, when studying multimorbidity from a qualitative perspective, it is important to take into account that diseases may also co-occur due to coincidence or selection bias. Second, several problems can arise by using hospital data for research purposes, because hospital records are not designed for research purposes but rather for patient care. Their diagnostic quality may vary depending on different hospitals, physicians, and clinical units. Moreover, hospital admissions are often selective on the basis of varying characteristics, such as severity of disease, associated medical conditions, and admissions policies that may vary from hospital to hospital. Third, the severity of diseases was not taken into account. However, the evaluation of disease severity is made difficult owing to several severity classification scales for the same disease and/or requirement of subjective judgement and clinical expertise. A fourth limitation is the issue of multiple comparisons between pairs of conditions. To minimize this problem, our analysis was limited to diseases with a prevalence $>5 \%$ in this sample of hospitalized elderly. Moreover, we discussed a possible association with inhospital mortality and adverse events only for those pairs of diseases that showed a significant association with a $p$ value of 0.001 or lower.

Although weighing co-morbidity in a clinical setting is limited by many serious methodological problems, the REPOSI study suggests that more efforts should be addressed at identifying subgroups of patients whose prognosis can be better defined on the simple basis of disease clustering. In spite of the fact that acute medical treatment is usually directed toward the nature and the severity of the main disease leading to hospitalization, it is no longer possible to overlook the confounding effect of multiple coexisting diseases in a clinical setting of geriatrics and internal medicine.

\section{Acknowledgments}

We thank Professor Giuseppe Licata, President of the Italian Society of Internal Medicine during the study period, for his help and encouragement.

\section{Author Disclosure Statement}

No competing financial interests exist.

\section{References}

1. van den Akker M, Buntinx F, Knottnerus JA. Comorbidity or multimorbidity: What's in a name? A review of literature. Eur J Gen Pract 1996;2:65-70.

2. Incalzi RA, Capparella O, Gemma A, Landi F, Bruno E, Di Meo F, Carbonin P. The interaction between age and comorbidity contributes to predicting the mortality of geriatric patients in the acute-care hospital. I Int Med 1997;242:291298.

3. Marengoni A, Cossi S, De Martinis M, Ghisla MK, Calabrese PA, Zanolini G, Leonardi R. Adverse outcomes in older hospitalized patients: the role of multidimensional geriatric assessment. Aging Clin Exp Res 2003;15:32-37.

4. Zekry D, Herrmann FR, Grandjean R, Vitale AM, De Pinho MF, Michel JP, Gold G, Krause KH. Does dementia predict adverse hospitalization outcomes? A prospective study in aged inpatients. Int J Geriatr Psychiatry 2009;24:283-291.

5. Geraci JM, Ashton CM, Kuykendall DH, Johnson ML, Wu L. In-hospital complications among survivors of admission for congestive heart failure, chronic obstructive pulmonary disease, or diabetes mellitus. J Gen Intern Med 1995;10: 307-14.

6. World Health Organization. International Classification of Diseases, Injuries, and Causes of Death, Ninth Revision (ICD-9). Geneva; 1987.

7. Boyd CM, Ritchie CS, Tipton EF, Studenski SA, Wieland D. From Bedside to Bench: Summary from the American Geriatrics Society/National Institute on Aging Research Conference on Comorbidity and Multiple Morbidity in Older Adults. Aging Clin Exp Res 2008;20:181-188.

8. World Health Organization. Guidelines for ATC Classification. WHO Collaborating Centre for Drug Statistics 
Methodology, Norway and Nordic Councils on Medicines. Sweden; 1990.

9. Verbrugge LM, Lepkowski JM, Imanaka Y. Comorbidity and its impact on disability. Milbank Q 1989;67:450-484.

10. van den Akker M, Buntinx F, Metsemakers JF, Roos $\mathrm{S}$, Knottnerus JA. Multimorbidity in general practice: prevalence, incidence, and determinants of co-occurring chronic and recurrent diseases. I Clin Epidemiol 1998;51: 367-375.

11. John R, Kerby DS, Hennessy CH. Patterns and impact of comorbidity and multimorbidity among communityresident American Indian elders. Gerontologist 2003;43:649660.

12. Marengoni A, Rizzuto D, Wang HX, Winblad B, Fratiglioni L. Patterns of chronic multimorbidity in the elderly population. J Am Geriatr Soc 2009;57:225-230.

13. Gijsen R, Hoeymans N, Schellevis FG, Ruwaard D, Satariano WA, van den Bos GA. Causes and consequences of comorbidity: A review. J Clin Epidemiol 2001;54:661-674.

14. Wolff JL, Starfield B, Anderson G. Prevalence, expenditures, and complications of multiple chronic conditions in the elderly. Arch Intern Med 2002;162:2269-2276.
15. Redelmeier DA, Tan SH, Booth GL. The treatment of unrelated disorders inpatients with chronic medical diseases. N Eng J Med 1998;338:1516-1520.

16. Boyd CM, Darer J, Boult C, Fried LP, Boult L, Wu AW. Clinical practice guidelines and quality of care for older patients with multiple comorbid diseases: implications for pay for performance. JAMA 2005;294:716-724.

\author{
Address correspondence to: \\ Alessandra Marengoni \\ Department of Medical and Surgery Sciences \\ University of Brescia \\ Division of Internal Medicine I, Spedali Civili \\ Piazzale Spedali Civili 1 \\ 25123 Brescia \\ Italy \\ E-mail: marengon@med.unibs.it \\ Received: November 29, 2009 \\ Accepted: March 19, 2010
}




\section{Appendix: Collaborators and Participating Units}

Alberto Tedeschi, Raffaella Rossio (Medicina Interna 2, Fondazione IRCCS Ospedale Maggiore, Milano); Guido Moreo, Barbara Ferrari (Medicina Interna 3, Fondazione IRCCS Ospedale Maggiore, Milano); Antonio Mammarella, Valeria Raparelli (Medicina Interna, Università La Sapienza, Roma); Stefania Rondinella, Iolanda Giannico (Medicina Metabolica, Università di Modena e Reggio Emilia); Leonardo Rasciti, Silvia Gualandi (Medicina Interna, Policlinico S. Orsola Malpighi, Bologna); Valter Monzani, Valeria Savojardo (Medicina d'Urgenza, IRCCS Fondazione Ospedale Maggiore, Milano); Giovanna Fabio, Silvia Colombo (Medicina Interna 1A, Fondazione IRCCS Ospedale Maggiore, Milano); Alessandra Quercioli, Alessandra Barreca (Medicina Interna 1, Università di Genova); Emanuele Durante-Mangoni, Daniela Pinto (Medicina Interna, Seconda Università di Napoli); Elena Incasa, Emanuela Rizzoli (Medicina Interna, Azienda USL, Ferrara); Massimo Vanoli, Gianluca Casella (Medicina Interna, Ospedale di Lecco, Merate); Giuseppe Musca, Olga Cuccurullo (Medicina Interna, P.O. Cetraro, ASP Cosenza); Giuseppe Famularo, Maria Rosaria Sajeva (Medicina Interna, Ospedale San Camillo Forlanini, Roma); Antonio Picardi, Dritan Hila (Medicina Clinica-Epatologia, Università Campus Bio-Medico, Roma); Renzo Rozzini, Alessandro Giordano (Fondazione Poliambulanza, Brescia); Antonio Bonelli, Gaetano Dentamaro (Medicina, Ospedale Madonna delle Grazie, Matera); Giulia Gobbo, Massimo Cazzaniga (Medicina Interna, IRCCS Policlinico San Donato, Università di Milano); Piergiorgio Gaudenzi, Lisa Giusto (Medicina ad Alta Rotazione, Azienda Ospedaliera Universitaria, Ferrara); Damiano Rizzoni, Luana Castoldi (Clinica Medica, Università di Brescia); Daniela Mari, Giuliana Micale (Medicina Generale ad indirizzo Geriatrico, IRCCS Istituto Auxologico Italiano, Milano); Emanuele Altomare, Gaetano Serviddio (Medicina Interna, Università di Foggia); Carlo Longhini, Cristian Molino (Clinica Medica, Azienda Mista Ospedaliera Universitaria Sant'Anna, Ferrara); Silvia Deidda, Luciana Maria Cuccuru (Clinica Medica, Azienda Mista Ospedaliera Universitaria, Sassari); Michela Quagliolo, Giuseppe Riccardo Centenaro (Medicina 1, Ospedale di Melegnano, Vizzolo Predabissi, Milano); Anna Laura Pasqui, Luca Puccetti (Medicina Interna, Azienda Ospedaliera Universitaria Le Scotte, Siena); Giampiera Bertolino, Piergiorgio Cavallo (Dipartimento di Medicina Interna, Fondazione IRCCS Policlinico San Matteo, Università degli Studi di Pavia); Daniele Bertolini, Nicola Lucio Liberato (Medicina Interna, Ospedale Carlo Mira, Casorate Primo, Pavia); Antonio Perciccante, Alessia Coralli (Medicina, Ospedale San GiovanniDecollato-Andisilla, Civita Castellana); Luigi Anastasio, Leonardo Bertucci (Medicina Generale, Ospedale Civile Serra San Bruno); Giancarlo Agnelli, Ana Macura (Medicina Interna e Cardiovascolare, Ospedale Santa Maria della Misericordia, Università di Perugia); Cosimo Morabito, Roberto Fava (Medicina, Ospedale Scillesi 
d'America, Scilla); Antonino Tuttolomondo, Riccardo Di Sciacca (Medicina Interna e Cardioangiologia, Università degli Studi di Palermo); Luisa Macchini, Anna Realdi (Clinica Medica 4, Università di Padova); Alessandra Fiorentini, Cristina Tofi (Geriatria, Ospedale di Montefiascone); Carlo Cagnoni, Antonio Manucra (UO Medicina e Primo Soccorso, Ospedale di Bobbio, Azienda USL di Piacenza); Giuseppe Romanelli, (UO Geriatria, Spedali Civili di Brescia); Michele Cortellaro, Maria Rachele Meroni (Medicina 3, Ospedale Luigi Sacco, Università di Milano); Dionigi Paolo Rossi, Carlo Vergani (Geriatria, Fondazione IRCCS Ospedale Maggiore e Università di Milano). 
